\title{
Outcomes of Fluoroscopically Guided Lumbar Transforaminal Epidural Steroid Injections in Degenerative Lumbar Spondylolisthesis Patients
}

\author{
Chaiwat Kraiwattanapong ${ }^{1}$, Supaporn Wechmongkolgorn ${ }^{2}$, Bangon Chatriyanuyok ${ }^{2}$, \\ Patarawan Woratanarat ${ }^{1}$, Umaporn Udomsubpayakul ${ }^{3}$, Pongsathorn Chanplakorn ${ }^{1}$, \\ Gun Keorochana ${ }^{1}$, Wiwat Wajanavisit ${ }^{1}$ \\ ${ }^{1}$ Department of Orthopaedics, Ramathibodi Hospital, Mahidol University, Bangkok, Thailand \\ ${ }^{2}$ Department of Nursing, Ramathibodi Hospital, Mahidol University, Bangkok, Thailand \\ ${ }^{3}$ Epidemiology Unit, Ramathibodi Hospital, Mahidol University, Bangkok, Thailand
}

Study Design: A prospective cohort.

Purpose: To report the short and long term outcomes of fluoroscopically guided lumbar transforaminal epidural steroid injection (TFESI) in degenerative lumbar spondylolisthesis (DLS) patients.

Overview of Literature: TFESI has been widely used for the treatment of lumbosacral radicular pains. However, to our knowledge, there has been no study which has evaluated the outcomes of TFESI in patients with DLS.

Methods: The DLS patients received fluoroscopically guided lumbar TFESI with $80 \mathrm{mg}$ of methylprednisolone and $2 \mathrm{~mL}$ of $1 \%$ lidocaine hydrochloride. Patients were evaluated by an independent observer before the initial injection, at 2 weeks, at 6 weeks, at 3 months, and at 12 months after the injections. Visual analog scale (VAS), Roland 5-point pain scale, standing tolerance, walking tolerance, and patient satisfaction scale were evaluated for outcomes.

Results: Thirty three DLS patients treated with TFESI, who were completely followed up, were included in this study. The average number of injections per patient was 1.9 (range from 1 to 3 injections per patient). Significant improvements in VAS and Roland 5-point pain scale were observed over the follow up period from 2 weeks to 12 months. However, the standing and walking tolerance were not significantly improved after 2 weeks. At 2 weeks, the patient satisfaction scale was highest, although, these outcomes declined with time. The DLS patients with one level of spinal stenosis showed significantly better outcome than the DLS patients with two levels of spinal stenosis. Five patients (13\%) underwent surgical treatment during the 3 to 12 months follow up.

Conclusions: TFESI provides short term improvements in VAS and Roland 5-point pain scale, standing tolerance, walking tolerance and patient satisfaction scale in DLS patients. In the long term, it improves VAS but limits the improvements in Roland 5-point pain scale, standing tolerance, walking tolerance and patient satisfaction scale.

Keywords: Degenerative lumbar; Spondylolisthesis; Epidural injection; Steroid; Outcome

Received Oct 22, 2012; Revised Jan 29, 2013; Accepted May 1, 2013

Corresponding author: Chaiwat Kraiwattanapong

Department of Orthopaedics, Ramathibodi Hospital,

Mahidol University, 270 Rama 6 road, Phayathai,Bangkok, Thailand 10400

Tel: +66818977640, Fax: +6622011599, E-mail: drchaiwat@yahoo.comOutcomes 


\section{Introduction}

Degenerative lumbar spondylolisthesis (DLS) is a common cause of low back pain, radiculopathy and neurogenic claudication in adults. The superior vertebra slides forward on the inferior resulting in a narrowed central canal and lateral recesses which lead to a wide range of clinical presentations. There are various types of nonoperative treatments for DLS such as medication, physical therapy, strengthening exercises or spinal manipulation. If the patients do not respond to such treatments within 4 to 6 weeks, they may benefit from an epidural steroid injection [1].

Epidural steroid injection has been used for the treatment of low back pains and leg pains for more than 50 years [2]. Several techniques and approaches have been developed to deliver the highest concentration of steroids to the target site of pathology without complications. Fluoroscopy with epidurography has been used to ensure the localization of needles within the epidural space. Even in experienced hands, the epidural steroid without the use of fluoroscopy or contrast media may be misplaced from the intended target in $30 \%$ of cases $[3,4]$. For lumbar spine, there are three approaches for epidural steroid injections, namely: transforaminal, interlaminar and caudal. According to systematic reviews, successful evidences of caudal epidural steroid injections in spinal stenosis are limited [5,6]. Fluoroscopic guided transforaminal epidural steroid injection (TFESI) has several advantages such as the accuracy, specific targeting, and safety with low volumes of injectate. For foraminal spinal stenosis, TFESI is usually selected initially and, for the stenosis of the upper lumbar central canal, the interlaminar epidural steroid injection is the first choice of treatment [7]. Currently, fluoroscopically guided TFESI is frequently being used for treatment of radicular pains in lumbar disc herniation, spinal stenosis and degenerative disc disease patients with various results. However, to our knowledge base on MEDLINE literature reviews, there has been no study which has exclusively evaluated the effectiveness of epidural steroid injections in patients with DLS.

The objectives of this study are to report the short- and long-term outcomes of fluoroscopically guided lumbar TFESI in DLS patients.

\section{Materials and Methods}

After approvals from our institutional review board, 38 DLS patients who underwent fluoroscopically guided lumbar TFESI during April 2009 to March 2010 were enrolled. There were 13 men and 25 women with an average age of 62 years (range, 44-81 years). All of the patients were provided with informed consents before being included in this study. The inclusion criteria were as follows: 1) patients with history of low back pain and unilateral radiating pain at least below the knee joint; 2) patients with slip grade 1 degenerative lumbar spondylolisthesis, visible on plain radiographs and one or two levels of neural compression found from the magnetic resonance imaging (MRI); 3) failures of conservative treatment by physiatrists for at least 6 weeks. These patients then received fluoroscopically guided lumbar TFESI according to the first author. The patients who had previous surgery at the lumbar spine, pars defects, previous history of epidural steroid injections, allergies to contrast media, gross neurological deficits, cauda equina syndrome or inflammatory joint diseases were excluded from the study. The patients were classified into 2 groups according to MRI findings with one or two levels of spinal canal stenosis. The patients with a single level of spinal stenosis received one level of fluoroscopically guided lumbar TFESI and the patients with two levels of stenosis, either one or two levels DLS, received two levels of fluoroscopically guided lumbar TFESI. The purpose of the different injection technique aimed to deliver the injectate as closely as possible to the pathology. Moreover, in severe lateral stenosis, the injectate could not be widely distributed.

The patients were evaluated by an independent observer before the initial injection (pre-injection), at 2 weeks, at 6 weeks, at 3 months, and at 12 months after the injections. The back pain, leg pain and neurological deficit of the patients were recorded. The visual analog scale (VAS), Roland 5-point pain scale, standing tolerance, walking tolerance and patient satisfaction scale were evaluated for the outcome measurements according to Botwin et al. (Table 1) [8]. At the follow up after injection, if the patients' symptoms were not improved, the patents were treated with reinjections or received operative treatments.

\section{Procedure description}

The patient was put in a prone position on a radiolu- 
Table 1. Five outcome measurements (From Botwin, et al. Am J Phys Med Rehabil 2002;81:898-905, with permission from Lippincott Williams \& Wilkins, Inc. [8])

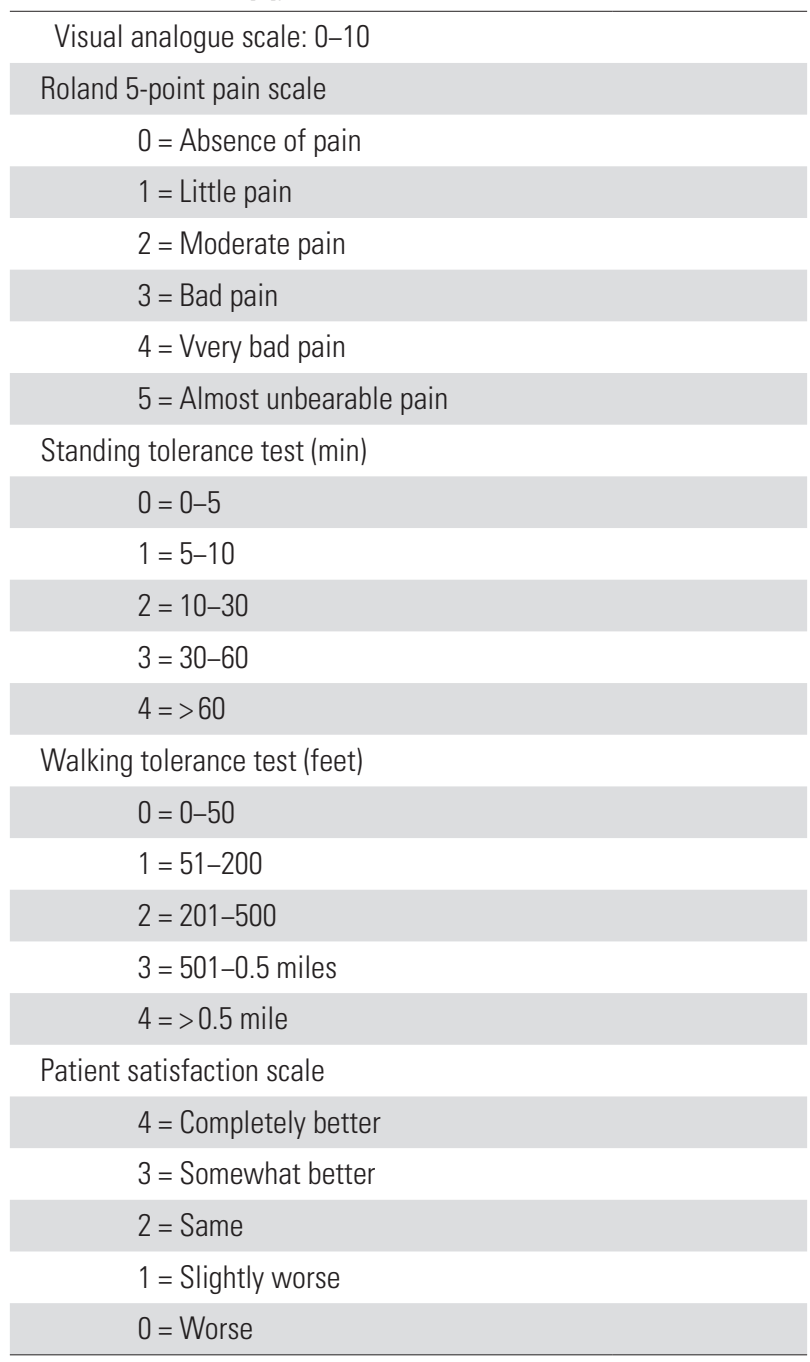

cent operating table. Then the C-arm fluoroscope was positioned to identify the level of spine and observe the foramen of the target level. The fluoroscopically guided lumbar TFESI level was performed using the preganglionic approach [9]. Local anesthetic (1\% lidocaine HCL [Xylocaine, AstraZeneca]) was then injected to the skin, subcutaneous tissues and the muscles where the needle is usually placed. Next, a 22-gauge spinal needle was advanced in the foramen underneath the pedicle using fluoroscopic guidance. Contrast media (Iopamiro, Bracco Industria Chemica SpA, Milan, Italy) was injected to confirm the epidural spreading (Fig. 1). After that, DepoMedral (Pharmacia \& Upjohn Company, New York, NY, USA) $2 \mathrm{~mL}$ ( $80 \mathrm{mg}$ ) with $1 \%$ lidocaine hydrochloride 2 $\mathrm{mL}$ were slowly injected. Finally, the patient was observed in the recovery room under standard protocol. If the patient was free from any complications then the patient was discharged.

\section{Statistical analysis}

Statistical analysis was performed using the STATA software ver. 10.0 (Statacorp Lp, College Station, TX, USA). The Wilcoxon rank sum test was used for the comparison in each parameter between pre-injection and post-injection outcome measurements at various time points. Additionally the comparison among outcome measurements between one level of stenosis and two levels of stenosis were determined using analysis of variance. The difference was considered significant if $p<0.05$.
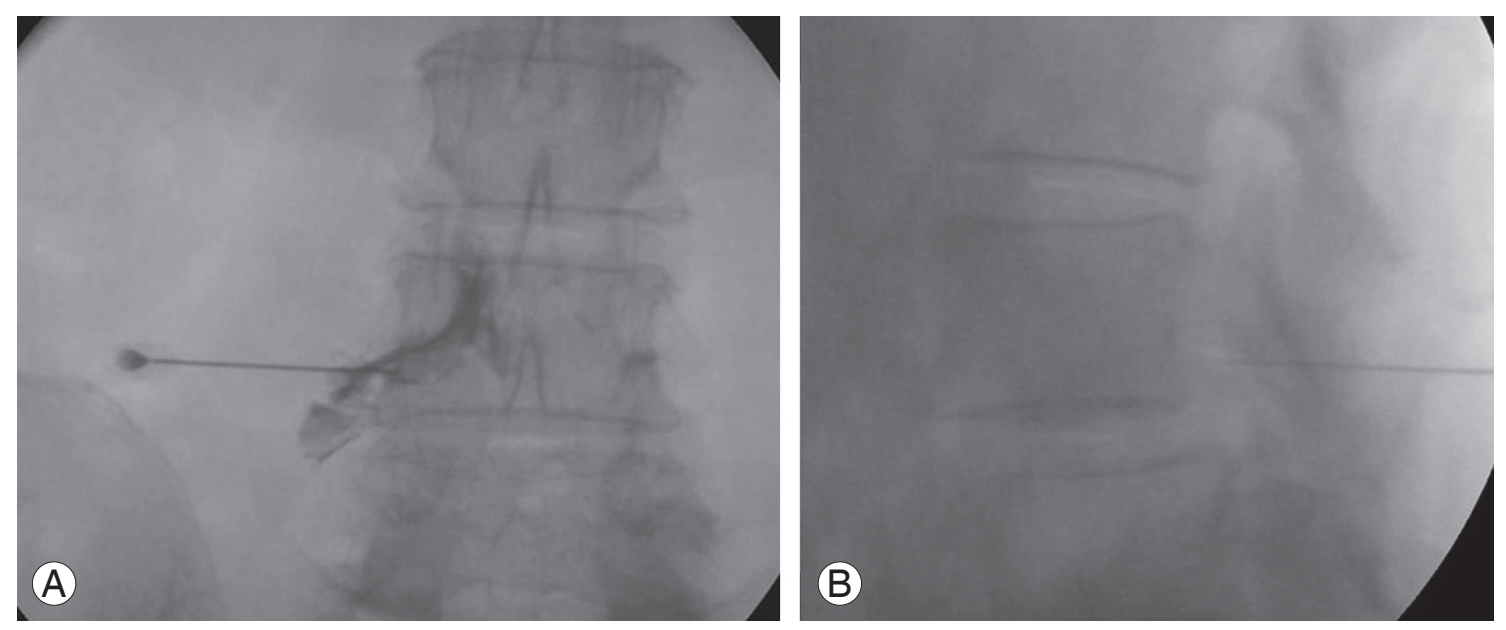

Fig. 1. Anteroposterior (A) and lateral (B) fluoroscopic projection of preganglionic transforaminal epidural steroid injection in lumbar spondylolisthesis patient showing contrast outlining L4 existing nerve root and spreading into epidural space. 
Table 2. Outcomes of TFESI in DLS patients pre-injection and at 2 weeks, 6 weeks, 3 months, and 12 months follow up assessments

\begin{tabular}{lccccc}
\multicolumn{1}{r}{ Outcomes } & Pre-injection & 2 wk & 6 wk & 3 mo & 12 mo \\
VAS & $6.06 \pm 1.12$ & $2.45 \pm 0.91^{\text {a) }}$ & $3.12 \pm 0.96^{\text {a) }}$ & $3.73 \pm 1.07^{\text {a) }}$ & $4.06 \pm 1.25^{\text {a) }}$ \\
Roland pain scale & $2.79 \pm 0.82$ & $1.52 \pm 0.51^{\text {a) }}$ & $2.12 \pm 0.7^{\text {a) }}$ & $2.33 \pm 0.65$ & $2.3 \pm 0.77$ \\
Standing tolerance & $1.82 \pm 0.88$ & $2.76 \pm 0.66^{\text {a) }}$ & $2.12 \pm 0.6$ & $1.9 \pm 0.61$ & $1.97 \pm 0.59$ \\
Walking tolerance & $1.55 \pm 0.83$ & $2.09 \pm 0.77^{\text {a) }}$ & $1.7 \pm 0.68$ & $1.76 \pm 0.61$ & $1.55 \pm 0.56$ \\
Patient satisfaction & - & $3.7 \pm 0.47$ & $3.45 \pm 0.51^{\text {bl }}$ & $3.36 \pm 0.49^{\text {b) }}$ & $3.27 \pm 0.63^{\text {b) }}$ \\
\hline
\end{tabular}

TFESI, transforaminal epidural steroid injection; DLS, lumbar spondylolisthesis; VAS, visual analog scale.

${ }^{\text {a) }}$ Statistically significant when compare to pre-injection $(p<0.05)$; ${ }^{b}$ Statistically significant when compare to patient satisfaction scale at 2 weeks post-injection $(p<0.05)$

Table 3. Outcomes of TFESI in DLS patients when compared between one level stenosis with two levels stenosis

\begin{tabular}{|c|c|c|c|c|c|c|c|c|}
\hline Outcomes & $\begin{array}{l}\text { Level of } \\
\text { stenosis }\end{array}$ & Pre-injection & 2 wk & 6 wk & $3 \mathrm{mo}$ & $12 \mathrm{mo}$ & $\begin{array}{l}p \text {-value } \\
\text { within }^{\text {a) }} \\
\text { groups }^{\text {a }}\end{array}$ & $\begin{array}{l}p \text {-value } \\
\text { between } \\
\text { groups }^{\text {b) }}\end{array}$ \\
\hline \multirow[t]{2}{*}{ VAS } & 1 & $6.00 \pm 1.14$ & $2.50 \pm 0.93^{\mathrm{c})}$ & $3.00 \pm 0.78^{c)}$ & $3.50 \pm 1.02^{c)}$ & $3.79 \pm 0.98^{c)}$ & $<0.001$ & \multirow[t]{2}{*}{0.012} \\
\hline & 2 & $6.22 \pm 1.09$ & $2.33 \pm 0.86^{c)}$ & $3.44 \pm 1.33^{\mathrm{cl}}$ & $4.33 \pm 1.00^{c)}$ & $4.78 \pm 1.64$ & $<0.001$ & \\
\hline \multirow[t]{2}{*}{ Roland } & 1 & $2.70 \pm 0.81$ & $1.54 \pm 0.51^{c)}$ & $2.00 \pm 0.59^{c /}$ & $2.21 \pm 0.66$ & $2.13 \pm 0.61^{c)}$ & $<0.001$ & \multirow[t]{2}{*}{0.004} \\
\hline & 2 & $3.00 \pm 0.87$ & $1.44 \pm 0.53^{c)}$ & $2.44 \pm 0.88$ & $2.67 \pm 0.50$ & $2.78 \pm 0.97$ & 0.001 & \\
\hline \multirow[t]{2}{*}{ Standing } & 1 & $1.91 \pm 0.93$ & $3.00 \pm 0.51^{c)}$ & $2.29 \pm 0.55$ & $2.13 \pm 0.54$ & $2.08 \pm 0.50$ & $<0.001$ & \multirow[t]{2}{*}{$<0.001$} \\
\hline & 2 & $1.56 \pm 0.73$ & $2.11 \pm 0.60$ & $1.67 \pm 0.50$ & $1.44 \pm 0.53$ & $1.67 \pm 0.71$ & 0.218 & \\
\hline \multirow[t]{2}{*}{ Walking } & 1 & $1.67 \pm 0.87$ & $2.29 \pm 0.75^{c)}$ & $1.92 \pm 0.58$ & $1.91 \pm 0.58$ & $1.67 \pm 0.56$ & 0.012 & \multirow[t]{2}{*}{$<0.001$} \\
\hline & 2 & $1.22 \pm 0.67$ & $1.56 \pm 0.53$ & $1.11 \pm 0.60$ & $1.33 \pm 0.50$ & $1.22 \pm 0.44$ & 0.510 & \\
\hline \multirow[t]{2}{*}{ Satisfaction } & 1 & - & $3.67 \pm 0.48$ & $3.54 \pm 0.51$ & $3.42 \pm 0.50$ & $3.38 \pm 0.58$ & 0.207 & \multirow[t]{2}{*}{0.058} \\
\hline & 2 & - & $3.78 \pm 0.44$ & $3.22 \pm 0.44$ & $3.22 \pm 0.44$ & $3.00 \pm 0.71^{d)}$ & 0.023 & \\
\hline
\end{tabular}

TFESI, transforaminal epidural steroid injection; DLS, lumbar spondylolisthesis; VAS, visual analog scale.

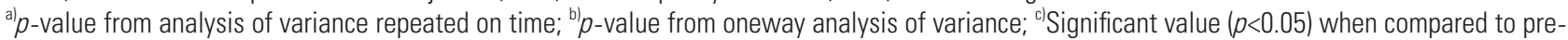
injection; ${ }^{\text {d) }}$ Significant value $(p<0.05)$ when compared to 2 weeks.

\section{Results}

Thirty eight DLS patients underwent fluoroscopically guided lumbar TFESI. The most common level of DLS was L4-L5 (69\%). All of the patients had experienced unilateral leg pains. Six of them (16\%) had no back pains. On initial neurological examination, there were no major neurological deficits found. Fourteen patients (37\%) have had mild extensor hallucis longus weaknesses. During 3 to 12 months of follow up, 5 patients underwent surgical treatments ( 4 due to intractable pains with severe neurogenic claudication, and 1 due to weakness of ankle dorsiflexion). The four patients with intractable pains responded well to TFESI during the first 6 weeks but later developed recurring pains. Three of them had DLS with 2 levels of spinal stenosis and, one of them had DLS with 1 level of spinal stenosis. A total of 33 patients completed follow ups at 1 year and their data were collected for analysis. There were 24 patients $(73 \%)$ with a one level stenosis and 9 patients (27\%) with two levels of stenosis. The average numbers of injection per patient was 1.9 (range, 1 to 3 injection per patient). There were no major complications reported from patients after the injection procedures. There were 3 patients who experienced worsening leg pains after injections but these symptoms resolved spontaneously after a few days.

\section{Comparison of pre-injection and post-injection}

The four outcome measurements: VAS, Roland 5-point pain scale, standing tolerance and walking tolerance of pre-injection were compared with post-injection at 2 weeks, 6 weeks, 3 months, and 12 months. Meanwhile, the patient satisfaction scale at 2 weeks post-injection was 
compared with 6 weeks, 3 months, and 12 months postinjection as shown in Table 2.

There were significant decreases in VAS scores between pre-injection and post-injection at 2 weeks, 6 weeks, 3 months and 12 months $(p<0.001)$. The Roland 5-point pain scale between pre-injection and post-injection were significantly improved at 2 weeks and 6 weeks $(p<0.001)$ respectively but were not significantly different from the pre-injection scores at 3 months and 12 months $(p=0.09$ and $p=0.091$, respectively).

For standing tolerance and walking tolerance, the significant difference was found only between pre-injection and post-injection at 2 weeks $(p<0.001)$. At 6 weeks, 3 months and 12 months, there were no significant improvements in standing tolerance and walking tolerance, $(p>0.05)$.

For the patient satisfaction scale, there were significant decreases in satisfaction between post-injection at 2 weeks and post-injection at 6 weeks, 3 months and 12 months ( $p=0.02, p=0.002$, and $p=0.005$, respectively).

\section{Comparison of one level of stenosis with two of levels stenosis}

Analysis of variance was used for comparing the outcome measurements after TFESI of one level stenosis DLS patients with two level stenosis DLS patients as shown in Table 3.

The VAS of both groups post-injection ( 2 weeks, 6 weeks, 3 months, and 12 months) were significantly lower than pre-injection $(p<0.001)$. When compared between groups, the VAS of one level of stenosis DLS patients post-injection was significantly lower than the VAS of two level of stenosis DLS patients $(p=0.012)$ as shown in Fig. 2.

The Roland 5-point pain scale of one level of steno-sis DLS patients post-injection (2 weeks, 6 weeks, and 12 months) were significantly lower than pre-injection $(p<0.001)$ while in two levels of stenosis DLS patients, the Roland 5-point pain scale post-injection was signifi-cantly lower than pre-injection ( $p=0.001)$ at 2 weeks. When compared between groups, the Roland 5-point pain scale of one level of stenosis DLS patients post-injection was significantly lower than the Roland 5-point pain scale of two levels of stenosis DLS patients $(p=0.012)$ as shown in Fig. 3.

The standing tolerance of one level of stenosis DLS patients post-injection at 2 weeks was significantly higher than pre-injection $(p<0.001)$ while in two levels of stenosis DLS patients, there was no significant differences in the standing tolerance between pre-injection and post-in-

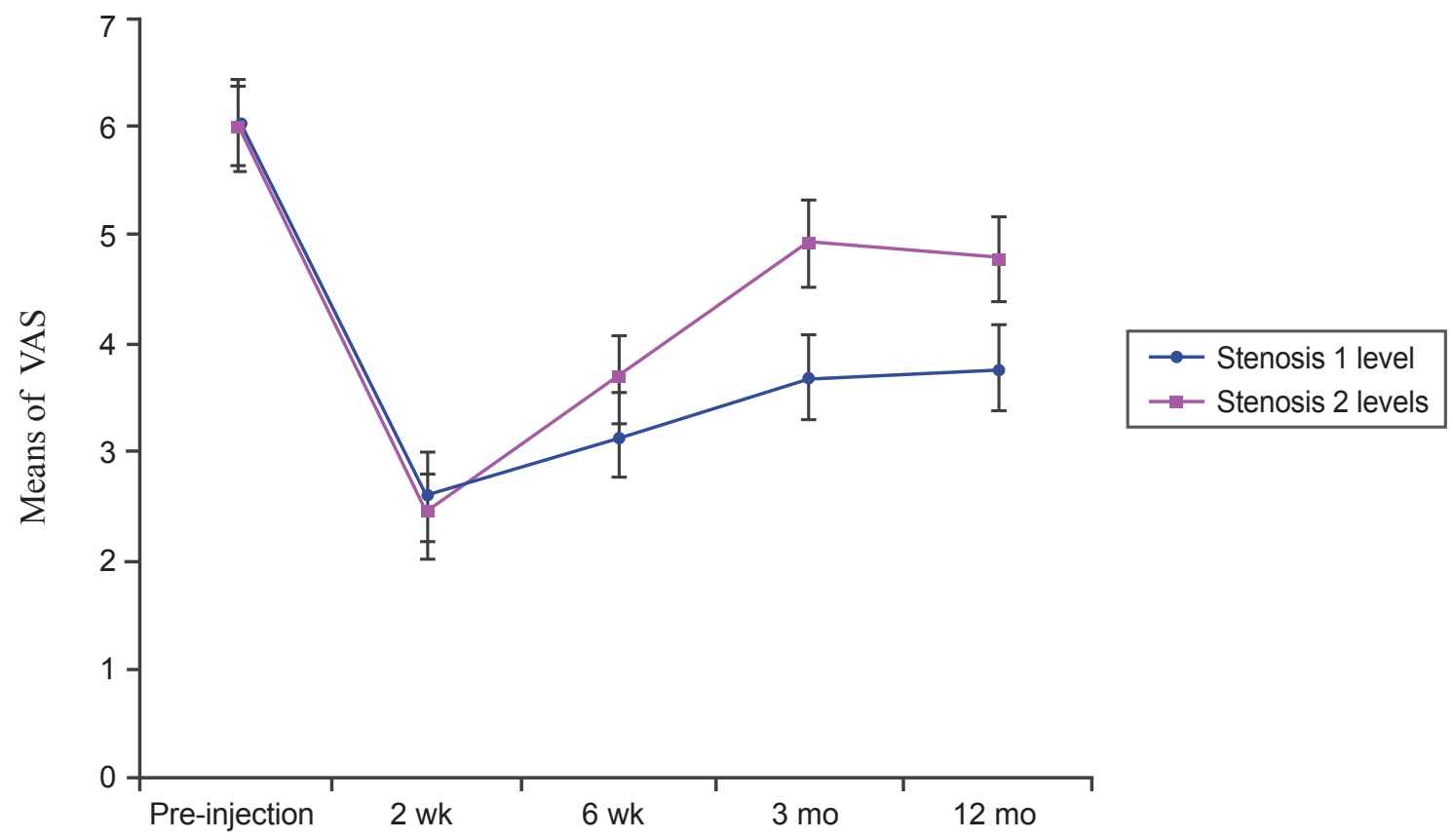

Fig. 2. Line graph showing the VAS of one level of stenosis DLS patients and two levels of stenosis DLS patients over time. There was a significant difference $(p=0.0124)$ between these two groups. VAS, visual analog scale; DLS, lumbar spondylolisthesis. 
jection ( $p=0.218$ ). When compared between groups, the standing tolerance of one level of stenosis DLS patients post-injection was significantly higher than the standing tolerance of two levels of stenosis DLS patients $(p<0.001)$ as shown in Fig. 4.
The walking tolerance of one of level stenosis DLS patients post-injection at 2 weeks was significantly higher than pre-injection $(p<0.012)$ while in two levels of stenosis DLS patients, there were no significant differences of the walking tolerance between pre-injection and post-

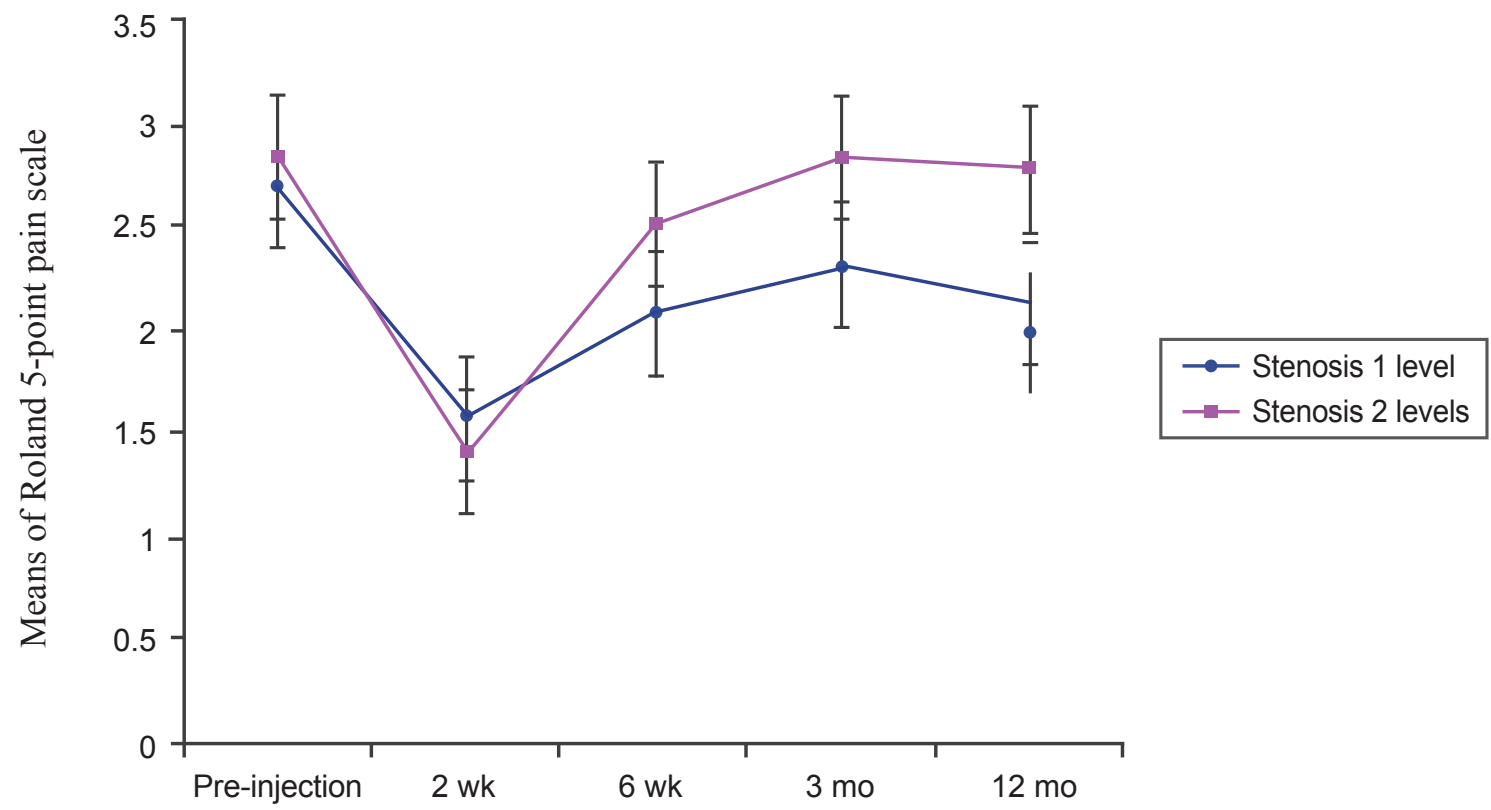

Fig. 3. Line graph showing the Roland 5-point pain scale of one level of stenosis DLS patients and two levels of stenosis DLS patients over time. There was a significant difference $(p=0.004)$ between these two groups. DLS, lumbar spondylolisthesis.

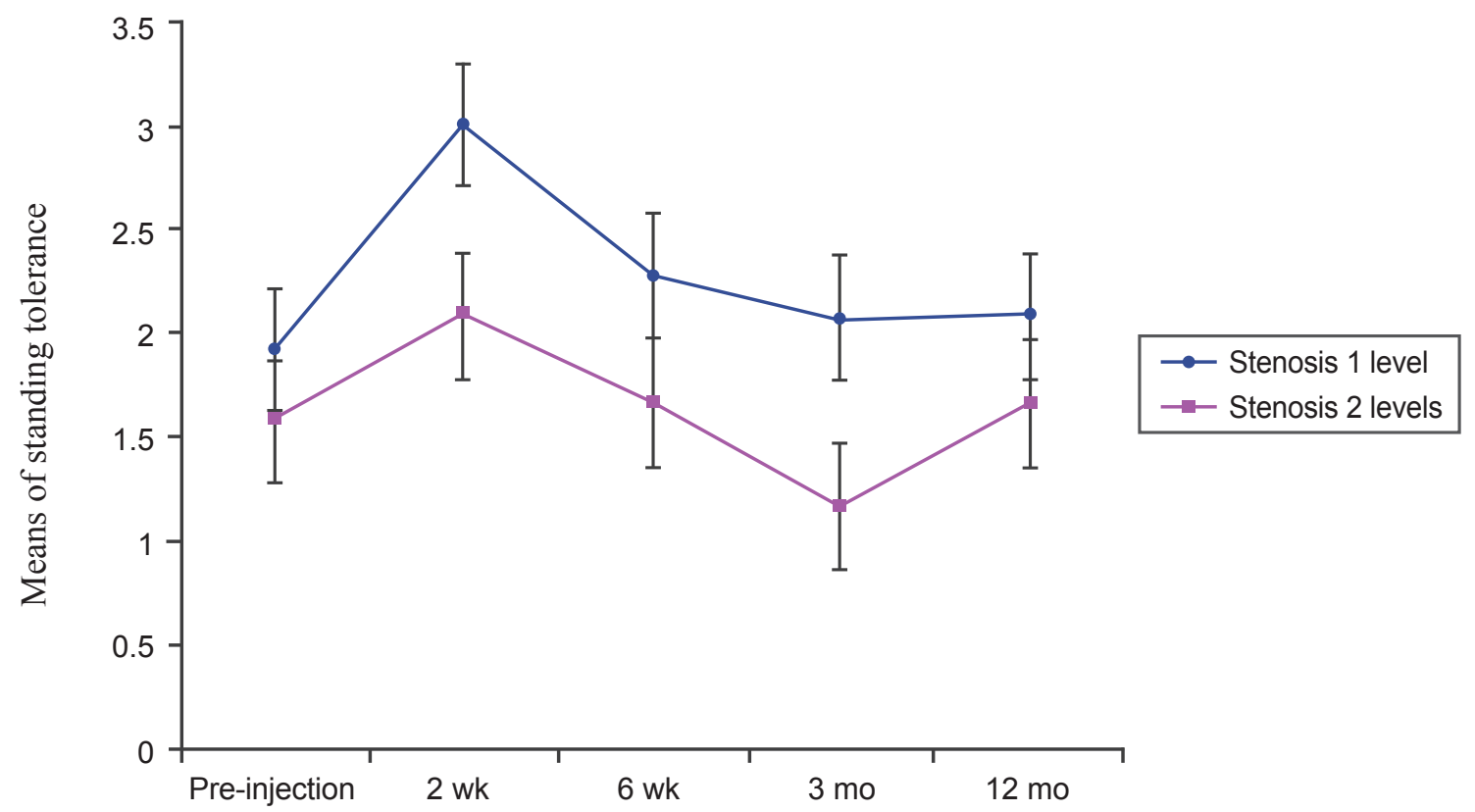

Fig. 4. Line graph showing standing tolerance of one level of stenosis DLS patients and two levels of stenosis DLS patients over time. There was a significant difference $(p<0.001)$ between these two groups. DLS, lumbar spondylolisthesis. 
injection $(p=0.510)$. When compared between groups, the walking tolerance of one level of stenosis DLS patients post-injection was significantly higher than the walking tolerance of two levels of stenosis DLS patients $(p<0.001)$ as shown in Fig. 5.
There were no significant differences of the patient satisfaction scale post-injection of both groups $(p=0.2072$ and $p=0.023$, respectively); except at 12 months, when the patient satisfaction scale was significantly lower than at 2 weeks $(\mathrm{p}<0.05)$. When compared between groups, there

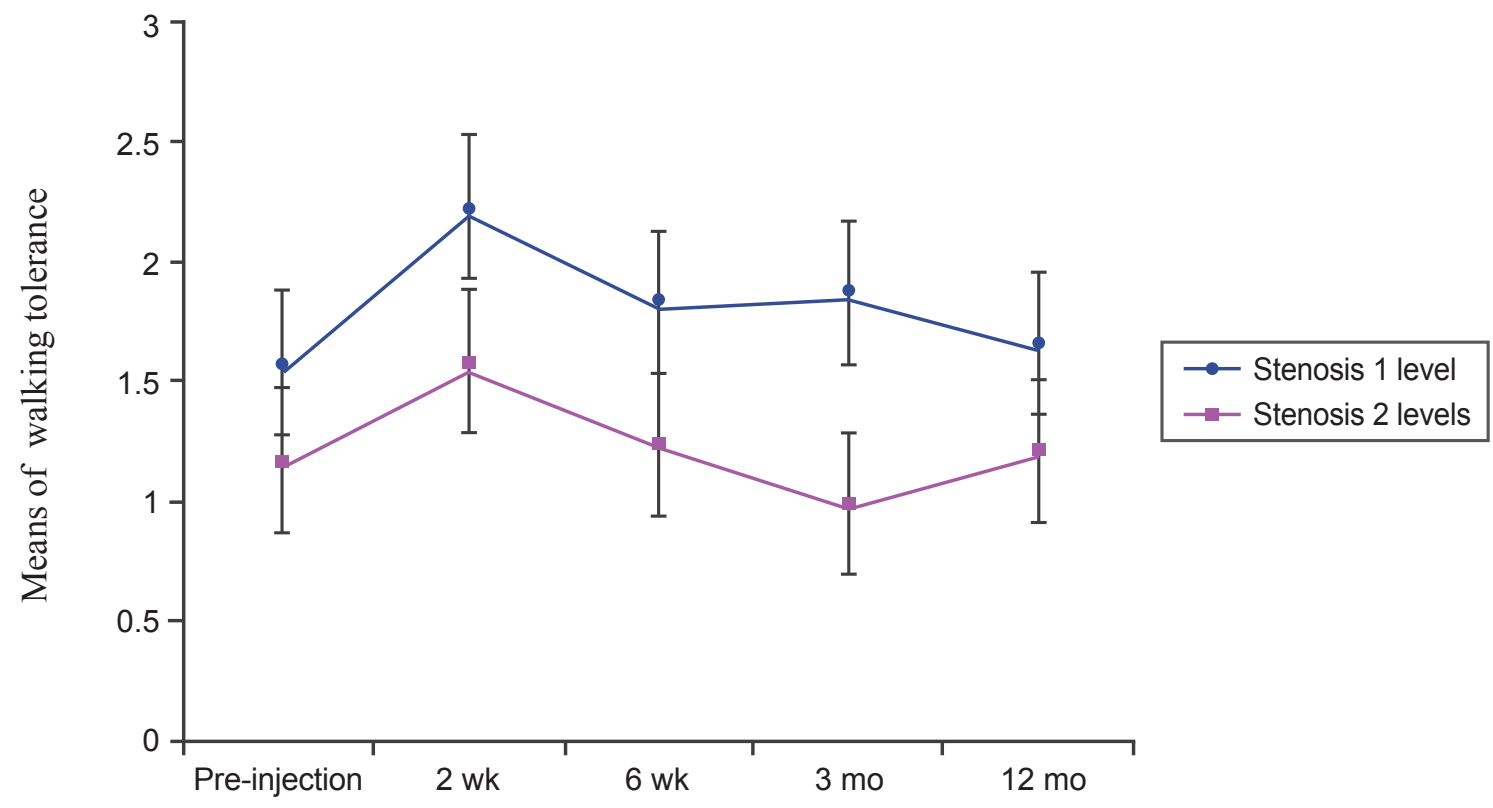

Fig. 5. Line graph showing walking tolerance of one level of stenosis DLS patients and two levels of stenosis DLS patients over time. There was a significant difference $(p<0.001)$ between these two groups. DLS, lumbar spondylolisthesis.

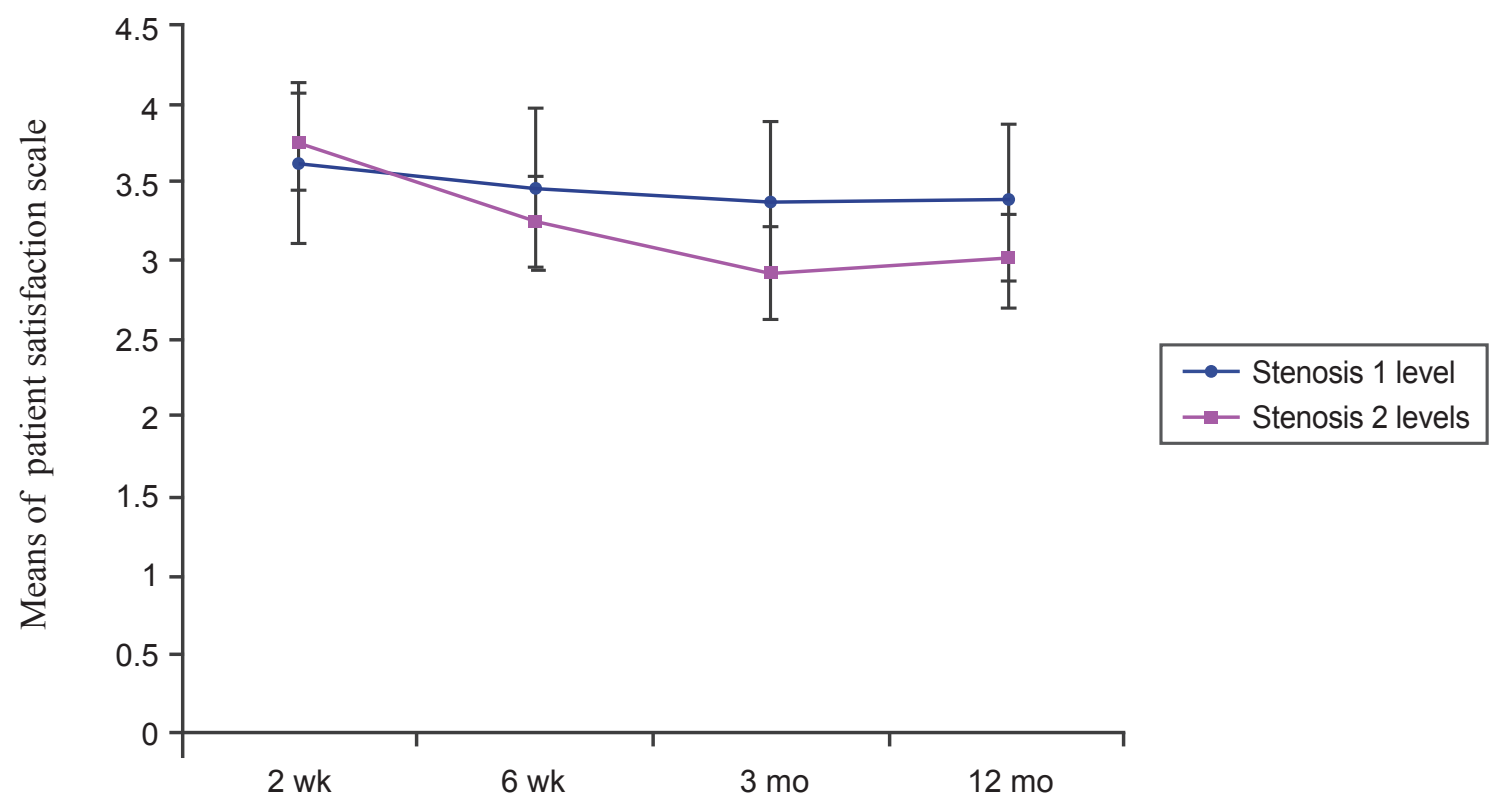

Fig. 6. Line graph showing the patient satisfaction scale of one level of stenosis DLS patients and two levels of stenosis DLS patients over time. There was no significant difference $(p=0.058)$ between these two groups. DLS, lumbar spondylolisthesis. 
was no significant difference in the patient satisfaction scale between both groups as shown in Fig. 6 .

\section{Discussion}

DLS is one of the common degenerative changes in the lumbar spine. The pathology and clinical presentation of DLS patients are different from the other degenerative changes of the lumbar spine. The pathology of DLS is overall problems of disc degeneration, facets arthrosis, central and lateral spinal canal stenosis with instability of ligaments and muscles $[10,11]$.

Epidural steroid injection has been used for treating lumbar disc herniation, lumbar disc degeneration and spinal stenosis.

There are several systematic reviews of TFESI in lumbar disc herniation which have shown favorable results especially in short term pain reliefs. However, in chronic axial low back pains from lumbar disc degeneration, the results of epidural steroid injections still lack well-designed studies [12-14]. For lumbar spinal stenosis, several studies have been published which evaluates epidural steroid injection with caudal epidural injection techniques [15-19], and TFESI techniques [8,20]. However, most of the population in the studies includes both spinal stenosis and DLS patients which cause inconsistent results, therefore, we chose to study the outcomes of epidural steroid injection exclusive to DLS patients.

There are many techniques of epidural steroid injection which have demonstrated different results. Schaufele et al. [21] reported that TFESI was superior to the interlaminar epidural steroid injections for treating lumbar disc herniation. Smith et al. [22] later reported no significant differences between TFESI and interlaminar epidural steroid injection for treating lumbar spinal stenosis. Abdi et al. [23] showed the evidence that lumbar TFESI is more effective than lumbar interlaminar epidural steroid injections. In the treatment of lumbar root pains, TFESI is strong for short term and moderate for long term efficacy. In contrast, the lumbar interlaminar epidural steroid injection is strong for short term reliefs and limited for long term efficacy. Botwin et al. [8] reported a prospective cohort study of 34 spinal stenosis patients who underwent fluoroscopically guided TFESI. They reported $75 \%$ of long term successful outcomes (at least $50 \%$ pain reduction post injection), a $64 \%$ improved walking tolerance and a $57 \%$ improved standing tolerance. With superior outcomes as mentioned above, we decided to use the TFESI technique for this study.

The results from our study showed that, TFESI significantly reduced VAS and Roland 5-point pain scores for both short term and long term follow ups. However, standing tolerance and walking tolerance only significantly improved in the short term (for 2 weeks), but in the long term there was no significant difference. The patient satisfaction scale for this procedure was highest at 2 weeks and declined with time.

These results may be explained by the multiple problems in the pathology of DLS as previously described. Corticosteroid has direct anesthetic effects by blocking the nociceptive c-fiber conduction and also inhibits phospholipase A2 activity which is found in injured nerves $[24,25]$. Therefore, it can reduce inflammatory pain which explains the reduction of VAS and Roland 5-point pain scores. However, neurogenic claudication and mechanical pain may not respond well with corticosteroid which is only shown by a fair improvement of standing tolerance and walking tolerance.

The outcome measurements were compared between one level of spinal stenosis DLS patients and two levels of spinal stenosis DLS patients after TFESI. All parameters indicated better outcome in one level of spinal stenosis patients than in two levels of spinal stenosis patients. These findings may be explained by the poor neural structures and physiology caused by double compression sites in neural elements.

The experiments by Olmarker and Rydevik [26] demonstrated that double level compressions induced more abnormal changes in nerve impulse conduction than one level. Takahashi et al. [27] studied the neural blood flow in pigs and reported that double level compressions of the cauda equina can induce impairment of blood flows at the compression site and intermediate nerve segments even at low pressures of compression which may cause dysfunctions of nerve impulse conduction. These studies support the poor results on two levels of spinal stenosis in DLS patients after TFESI.

Our study has some limitations. First, the sample size was small. We tried to select only patients with DLS who had prominent unilateral leg pains. For this homogenous group of patients, we avoided using various techniques of injections. However, some patients had DLS with one level stenosis, while the others had DLS with two levels of spinal stenosis. There has been no study for the outcomes 
of one needle TFESI as compared with two needles TFESI. For the benefit of the patients with two levels of spinal stenosis, we used two needles TFESI as mentioned in the methods. Another limitation was the numbers of injections were not the same for every patient. Repeated injections were done in patients with symptom recurrences.

\section{Conclusions}

The fluoroscopically guided lumbar TFESI is able to reduce VAS and Roland 5-point pain scale and improves standing tolerance and walking tolerance in the short term for DLS patients. For long term results, it reduces VAS but the improvement in standing tolerance and walking tolerance are limited. In addition, DLS patients with one level of spinal stenosis showed significantly better outcomes than DLS patients with two levels of spinal stenosis.

\section{Conflict of Interest}

No potential conflict of interest relevant to this article was reported.

\section{References}

1. Vibert BT, Sliva CD, Herkowitz HN. Treatment of instability and spondylolisthesis: surgical versus nonsurgical treatment. Clin Orthop Relat Res 2006;443:222-7.

2. Lutz GE, Vad VB, Wisneski RJ. Fluoroscopic transforaminal lumbar epidural steroids: an outcome study. Arch Phys Med Rehabil 1998;79:1362-6.

3. Weinstein SM, Herring SA, Derby R. Contemporary concepts in spine care. Epidural steroid injections. Spine (Phila Pa 1976) 1995;20:1842-6.

4. Stewart HD, Quinnell RC, Dann N. Epidurography in the management of sciatica. Br J Rheumatol 1987;26:424-9.

5. Abdi S, Datta S, Lucas LF. Role of epidural steroids in the management of chronic spinal pain: a systematic review of effectiveness and complications. Pain Physician 2005;8:127-43.

6. Boswell MV, Trescot AM, Datta S, et al. Interventional techniques: evidence-based practice guidelines in the management of chronic spinal pain. Pain Physician 2007;10:7-111.
7. Lee JW, Myung JS, Park KW, et al. Fluoroscopically guided caudal epidural steroid injection for management of degenerative lumbar spinal stenosis: short-term and long-term results. Skeletal Radiol 2010;39:691-9.

8. Botwin KP, Gruber RD, Bouchlas CG, et al. Fluoroscopically guided lumbar transformational epidural steroid injections in degenerative lumbar stenosis: an outcome study. Am J Phys Med Rehabil 2002;81:898905.

9. Jeong HS, Lee JW, Kim SH, Myung JS, Kim JH, Kang HS. Effectiveness of transforaminal epidural steroid injection by using a preganglionic approach: a prospective randomized controlled study. Radiology 2007;245:584-90.

10. Sengupta DK, Herkowitz HN. Degenerative spondylolisthesis: review of current trends and controversies. Spine (Phila Pa 1976) 2005; 30(6 Suppl):S71-81.

11. Kalichman L, Hunter DJ. Diagnosis and conservative management of degenerative lumbar spondylolisthesis. Eur Spine J 2008;17:327-35.

12. DePalma MJ, Slipman CW. Evidence-informed management of chronic low back pain with epidural steroid injections. Spine J 2008;8:45-55.

13. Southern D, Lutz GE, Cooper G, Barre L. Are fluoroscopic caudal epidural steroid injections effective for managing chronic low back pain? Pain Physician 2003;6:167-72.

14. Buttermann GR. The effect of spinal steroid injections for degenerative disc disease. Spine J 2004;4:495-505.

15. Manchikanti L, Cash KA, McManus CD, Pampati V, Abdi S. Preliminary results of a randomized, equivalence trial of fluoroscopic caudal epidural injections in managing chronic low back pain: Part 4--Spinal stenosis. Pain Physician 2008;11:833-48.

16. Botwin K, Brown LA, Fishman M, Rao S. Fluoroscopically guided caudal epidural steroid injections in degenerative lumbar spine stenosis. Pain Physician 2007;10:547-58.

17. Barre L, Lutz GE, Southern D, Cooper G. Fluoroscopically guided caudal epidural steroid injections for lumbar spinal stenosis: a restrospective evaluation of long term efficacy. Pain Physician 2004;7:187-93.

18. Delport EG, Cucuzzella AR, Marley JK, Pruitt CM, Fisher JR. Treatment of lumbar spinal stenosis with epidural steroid injections: a retrospective outcome study. Arch Phys Med Rehabil 2004;85:479-84. 
19. Ciocon JO, Galindo-Ciocon D, Amaranath L, Galindo D. Caudal epidural blocks for elderly patients with lumbar canal stenosis. J Am Geriatr Soc 1994;42:5936.

20. Cooper G, Lutz GE, Boachie-Adjei O, Lin J. Effectiveness of transforaminal epidural steroid injections in patients with degenerative lumbar scoliotic stenosis and radiculopathy. Pain Physician 2004;7:311-7.

21. Schaufele MK, Hatch L, Jones W. Interlaminar versus transforaminal epidural injections for the treatment of symptomatic lumbar intervertebral disc herniations. Pain Physician 2006;9:361-6.

22. Smith CC, Booker T, Schaufele MK, Weiss P. Interlaminar versus transforaminal epidural steroid injections for the treatment of symptomatic lumbar spinal stenosis. Pain Med 2010;11:1511-5.

23. Abdi S, Datta S, Trescot AM, et al. Epidural steroids in the management of chronic spinal pain: a system- atic review. Pain Physician 2007;10:185-212.

24. Olmarker K, Byrod G, Cornefjord M, Nordborg C, Rydevik B. Effects of methylprednisolone on nucleus pulposus-induced nerve root injury. Spine (Phila $\mathrm{Pa}$ 1976) 1994;19:1803-8.

25. Johansson A, Hao J, Sjolund B. Local corticosteroid application blocks transmission in normal nociceptive C-fibres. Acta Anaesthesiol Scand 1990;34:335-8.

26. Olmarker K, Rydevik B. Single-versus double-level nerve root compression. An experimental study on the porcine cauda equina with analyses of nerve impulse conduction properties. Clin Orthop Relat Res 1992;(279):35-9.

27. Takahashi K, Olmarker K, Holm S, Porter RW, Rydevik B. Double-level cauda equina compression: an experimental study with continuous monitoring of intraneural blood flow in the porcine cauda equina. J Orthop Res 1993;11:104-9. 